\title{
Islam y participación política en Mali: las elecciones presidenciales de 2013 \\ Islam and political participation in Mali: 2013 presidential election
}

David NIEVAS BULLEJOS

Investigador

Taller de Estudios Internacionales Mediterráneos

davidnievasb@gmail.com

Recibido 26/10/2017. Revisado y aprobado para publicación 20/11/2017

Para citar este artículo: David Nievas Bullejos (2017), "La participación política del islam en las elecciones presidenciales de 2013 en Mali" en Revista de Estudios Internacionales Mediterráneos, 23, 77-96.

Para acceder a este artículo: https://doi.org/10.15366/reim2017.23.005

\section{Resumen}

Desde la década pasada, se ha observado que han ido teniendo lugar en Mali algunas transformaciones sociales que podríamos sintetizar en una mayor presencia del islam y lo islámico en la esfera pública, empezando por los líderes religiosos. Estos, algunos de ellos grandes figuras carismáticas, han pasado a abordar en sus sermones cuestiones de debate sobre asuntos de índole social y nacional y han mostrado interés en el campo político. Dicho interés se ha traducido en la participación en el proceso electoral del año 2013, con la creación de un movimiento políticoreligioso, Sabati 2012. Aunque el objetivo de dicho movimiento no sea la toma del poder político ni la imposición de un modelo de Estado distinto, se puede afirmar que la participación en política de movimientos con referencias islámicas, así como líderes islámicos, ya no es solo una posibilidad entre otras sino un recorrido que ha comenzado a ser transitado en Mali.

Palabras clave: Mali/ islam/ participación política/ elecciones/ islamismo.

\section{Abstract}

Since the last decade, Mali has undergone a number of social transformations that indicate the increasing influence of Islam and Islamic values in the public sphere, the first obvious sign being the presence of religious leaders. These leaders, some of them important and charismatic figures, have begun to address matters of social and national importance in their sermons and have shown an interest in the political sphere, represented by their participation in the 2013 electoral process after the creation of the Sabati 2012 political-religious movement. Although the aim of this movement is 
not the seizure of political power or the imposition of a different state model, participation in politics on the part of movements with Islamic references and Islamic leaders is now an established path in Mali.

Keywords: Mali/ Islam/ political participation/ elections/ Islamism

\section{Introducción}

En los últimos años, Mali ha sido objeto de interés de los medios de comunicación y de los foros gubernamentales y académicos por la inestabilidad que desde allí ha emanado. Al tiempo que se iba constatando el incremento de la presencia yihadista, en Mali han ido teniendo lugar algunas transformaciones sociales que podríamos sintetizar en una mayor presencia del islam y lo islámico en la esfera pública, empezando por la presencia de los líderes religiosos. Los observadores y analistas, tanto dentro como fuera de Mali (Laremont y Gregorian, 2006) ${ }^{1}$, plantearon la posibilidad de que esta tendencia diera lugar al nacimiento de un movimiento de corte islamista o de grupos islámicos que tuvieran como objetivo la toma del poder político y la consecuente islamización del Estado, y que ello favoreciese la expansión de una ideología militante y yihadista entre sus ciudadanos caracterizados, hasta el momento, por un islam imbuido de "prácticas sufíes sincréticas, tolerancia y moderación" (Cormack, 2005). Así, al interés por el aumento del fenómeno yihadista en la región, se sumó el interés por lo religioso en un sentido más amplio (Dickson, 2005). El temor a que grupos organizados compitieran en política y en la calle por llegar al poder, como sucediera en Egipto o Túnez, puso en tensión a los observadores y a parte de la academia, al interpretar el aumento de la presencia del islam y de sus líderes como una señal de la islamización de la política, o, incluso, de radicalización de su sociedad.

Nuestro interés en esta temática ha sido generado tras la constatación de que la región estaba escasamente explorada entre los académicos e investigadores en lengua castellana, siendo prácticamente un territorio virgen para los estudios islámicos. Pensamos que una investigación sobre las dinámicas políticas del islam en Mali no solo tendría calado local, sino también regional e internacional, y podría ser interesante y una buena aportación al ámbito de estudio del islamismo y la participación política de actores islámicos. Así, este artículo aspira al mejor conocimiento del islam contemporáneo en dicha región y al estudio de la intersección de la religión y la política en las sociedades africanas contemporáneas ${ }^{2}$.

La atención en la última década sobre el islam en Mali radica en el potencial político de determinados líderes y asociaciones y en la consideración de que la presencia de un islam de corte radical y acción violenta pueda añadir problemas e inestabilidad a tan complejo territorio. La

\footnotetext{
${ }^{1}$ Este discurso también destacaba en los intercambios informales con investigadores locales, observadores electorales procedentes de la región, funcionarios locales que podríamos definir como "laicos" y funcionarios extranjeros destinados en el país con los que conversamos durante nuestra estancia en el país en el verano de 2013 con motivo de las elecciones presidenciales.

${ }^{2}$ El islam subsahariano representaba en el año 2010 alrededor de 240 millones de individuos (Pew Research Center, 2011) -quizás más actualmente - distribuidos en una amplia zona geográfica que engloba el continente africano al sur del Sáhara. De todos los fieles musulmanes en el mundo, este volumen de creyentes en el África Subsahariana representa un sexto de la población musulmana mundial. Esto es el resultado de la historia del islam en el continente. Unas poblaciones africanas musulmanas que quizá estén poco investigadas por haber estado el foco en el Norte de África y Oriente Medio, y una historia islámica poco estudiada dentro del campo de los estudios islámicos, que se remonta al siglo IX con el contacto de las poblaciones de África del Oeste con comerciantes musulmanes del Norte de África.
} 
historia reciente del país africano muestra que los jóvenes malienses no han sido inmunes a procesos de radicalización, incluyendo la integración en grupos armados de inspiración salafista y proclives a la acción violenta. Por otro lado, tampoco se puede descartar la emergencia de un islam que intente hacerse con el poder político e islamizar la sociedad como en otros países del mundo islámico. Este trabajo, a través de la revisión del caso de la creación de una asociación político-religiosa llamada Sabati 2012, pretende aportar argumentos para el debate sobre estas cuestiones con la intención de aclarar y comprender la naturaleza contemporánea de los grupos musulmanes interesados en política presentes en Mali. Para ello, hemos basado nuestra investigación en entrevistas realizadas sobre el terreno a una diversidad de actores relacionados con la temática indagada. Este artículo se fundamenta en entrevistas a líderes islámicos, como predicadores, miembros de la asociación Alto Consejo Islámico de Mali y miembros del movimiento Sabati 2012, entre el año 2014 y 2016 en Bamako, así como también se basa en la observación electoral in situ de las elecciones presidenciales del verano de $2013^{3}$ y distintas observaciones sobre el terreno a través de estancias cortas en Bamako entre el año 2014 y 2016.

El caso de Mali plantea ciertos debates de relevancia para comprender las transformaciones de la sociedad y del ámbito religioso en los distintos países de la región del Sahel. Mali es una democracia con una población íntegramente musulmana y con una organización estatal laica, lo que puede aportar interesantes discusiones en la diversa casuística del islamismo y de la participación política del islam que existe en el mundo islámico. Pese al carácter laico del Estado, hoy en día las fuerzas religiosas son de gran importancia en la sociedad maliense y su influencia se hace patente en la política nacional, materializado por la creación de la asociación Sabati 2012. Desde la llegada de las libertades y la democracia en los años 90, el islam ha experimentado un crecimiento en cuanto a su visibilidad y una importancia mayor en la sociedad (Holder, 2009) y entre los practicantes musulmanes hay un mayor interés y compromiso por practicar el islam correctamente, reafirmando su validez como cualquier otro islam practicado en otros lugares. Si no se puede entender la construcción del Estado maliense sin tener en cuenta el papel del islam, tampoco podemos analizar la realidad actual del país sin atender de modo cuidadoso a la presencia del islam en los acontecimientos políticos ${ }^{4}$.

La creación del movimiento político-religioso Sabati 2012 para las elecciones presidenciales que iban a tener lugar en el año 2012 - que finalmente fueron aplazadas al verano de 2013- ha sido la consolidación de la tendencia de la participación en política de los líderes religiosos y de asociaciones religiosas que venía teniendo lugar desde hacía unos años en Mali. Ante el retraimiento del Estado en diversos dominios y la percepción del fracaso de la clase política maliense ante los graves desafíos que ha enfrentado el país, líderes islámicos e individuos cercanos a los círculos islámicos creyeron que los musulmanes malienses debía movilizarse para pesar sobre el destino político del país y contribuir a corregir las deficiencias políticas encontradas en los años recientes y erradicar ciertas agresiones percibidas a los valores religiosos de la

\footnotetext{
${ }^{3}$ Llevé a cabo la observación electoral de las elecciones presidenciales de julio y agosto del año 2013 en el marco de la misión de observación del Observatorio Político y Electoral del Mundo Árabe y Musulmán (OPEMAM) de la Universidad Autónoma de Madrid y gracias a la financiación de la Agencia Española de Cooperación Internacional al Desarrollo.

${ }^{4}$ La relación entre el islam y la política en el África Subsahariana no es un ámbito muy desarrollado por los estudios africanos.
} 
sociedad por algunos sectores políticos laicistas. Para ello, la creación del movimiento Sabati y su participación en los comicios presidenciales a través del apoyo público a un candidato podría hacer efectivo el interés de algunos sectores islámicos en la política y canalizarlo en un resultado político.

\section{La participación política e institucional del islam en el Mali democrático}

Habiendo sido inédito anteriormente, es a partir del año 2000 que varios autores han observado una creciente difusión del islam y de su importancia en la esfera pública en Mali, con la creación incluso de una "esfera islámica", y han reflexionado sobre el proceso de incorporación de algunos actores del islam maliense en política (Brenner, 2001; Schulz, 2003; Miles, 2007; Otayek y Soares, 2007; Holder, 2009).

Tras 23 años de mandato autoritario del militar Mussa Traoré (1968-1991), las fuerzas prodemocráticas y una parte del ejército pusieron fin al régimen del militar e iniciaron una transición hacia la democracia y el multipartidismo que fue modélica en África del Oeste. Las autoridades transitorias organizaron una Conferencia Nacional en la que diferentes sectores políticos y sociales del país debatieron sobre los términos de construcción del nuevo país y de la nueva Constitución. Un momento importante fueron las deliberaciones en torno a la relación del Estado con la religión, dando como resultado el acuerdo sobre la laicidad del Estado (Wing, 2008). Partiendo de la perspectiva planteada por Leininger $(2010)^{5}$ basada en el estudio del proceso de deliberaciones políticas en la transición a la democracia, podría afirmarse que las dinámicas de interferencia del islam en el campo político objeto de análisis en este artículo vendrían determinadas por la poca influencia de los actores islámicos en la transición, que dio como resultado el establecimiento de unas relaciones Estado-religión continuadores del régimen autoritario anterior. Leininger explica esta falta de presencia por el marco laico del Estado maliense y por el débil proceso de institucionalización del islam en primer lugar.

Sin embargo, a principios de los años 2000 se sentaron las bases para la creación de una asociación de representantes musulmanes de todas las tendencias para facilitar su interlocución con el Estado. La necesidad de generar un modelo de interlocución era una consecuencia de la liberalización del espacio público que había tenido lugar en los años 90 que dio como resultado una explosión del asociacionismo islámico y la emergencia de figuras islámicas carismáticas gracias a la utilización de las nuevas tecnologías.

En su trabajo, Leininger (2010) analiza el papel de los actores islámicos ${ }^{6}$ en la consolidación de la joven democracia maliense, señalando que la presencia de estos actores produce dos efectos: un reforzamiento de la democracia, por un lado, a la vez que un menoscabo de la misma por otro. Ante la pluralización del campo islámico la autora analiza que el Estado ve la necesidad de

\footnotetext{
5 "The Diverse Role of Muslim Actors in Mali's Democratic Consolidation: Fostering Plurality to Inhibiiting Strong State Institutions", presentado en el taller "Religious Actors in Democratization Processes: Evidence from the five Muslim Democracies" que tuvo lugar en la Universidad de Princeton (EEUU) (Leininger, 2010).

${ }^{6}$ Leininger (2010: 25) engloba como actores islámicos a una serie de individuos y personalidades islámicas organizadas o no que se reclaman de una identidad islámica, que desgrana en: panorama heterogéneo de organizaciones islámicas, asociaciones islámicas con intereses políticos, representación formal de la comunidad islámica en el campo político maliense a través del ACIM, centros sufíes, comités de mezquitas, autoridades islámicas carismáticas, ONGs y organizaciones especializadas y descentralizadas. No obstante, no señala ningún ejemplo de las categorías que menciona ni señala que muchas de ellas están interconectadas unas con otras.

80
} 
agruparlo en un único interlocutor, al que, dotándole de capacidad de interlocución acaba concediéndole "espacio para maniobrar y para hacer de ellos una parte institucionalizada de la política nacional" (Leininger, 2010: 18). Así, los actores islámicos, representados en la asociación Alto Consejo Islámico de Mali (ACIM), comenzaron a tener un papel en la política del país, especialmente por la actuación de su presidente, al que Leininger señala como el mayor hacedor de dichas dinámicas. La politóloga apunta que las demandas y reivindicaciones de los actores islámicos que no fueron escuchadas durante la transición maliense no desaparecieron, sino que habrían reaparecido ahora, canalizadas a través de la asociación que aspira a representar todas las sensibilidades de los musulmanes, el ACIM. Aceptando el juego democrático, éstos han contribuido a la "pluralización de la joven democracia maliense" y han contribuido al fortalecimiento de la sociedad civil, aspecto indispensable para un sistema democrático (Leininger, 2016: 22). No obstante, la autora concede a los actores islámicos una influencia negativa en la consolidación de la democracia. Leininger analiza las tomas de posición de estos actores respecto a "ciertas libertades, especialmente en el ámbito de la ley de familia, y más en general, igualdad de género" (Leininger, 2016: 22) como un menoscabo democrático que impide la consolidación total de la democracia desde el campo "extraparlamentario".

Los métodos de participación política de los actores islámicos que analizamos en este trabajo completan los análisis de Leininger (2010 y 2016) y de Künkler y Leininger (2009). Künkler y Leininger (2009) afirman que la capacidad de movilización de los actores islámicos en Mali era reducida, como continuación de la misma situación de la época autoritaria del presidente Mussa Traoré (1968-1991), donde no habían podido ser un actor unificado y con presencia suficiente para incluir sus valoraciones. "Sobre todo, los heterogéneos actores religiosos de Mali poseen una baja capacidad de movilización" (Künkler y Leininger, 2009: 1080), afirmación que contrasta con las demostraciones de movilización popular que en el mismo año de la publicación del texto, 2009, tuvieron lugar en Bamako y otros lugares del país. Para Leininger este hecho es algo importante porque "por primera vez en la historia de la República de Mali, organizaciones islámicas, en particular el ACIM, organizaron protestas públicas contra las políticas del Gobierno" (Leininger, 2010: 23). Si tomamos como referencia estas afirmaciones, basadas en el análisis de la influencia política de los actores islámicos, debemos de indicar que después de las infructuosas formas de influir en la transición de principios de los años 90, los primeros intentos fueron en el cambio de milenio en las elecciones del año 2002, con poco éxito de nuevo hasta llegar al episodio de las protestas contra el Código de familia y de las personas en 2009 para terminar la línea ascendente en las elecciones del año 2013, en las que, esta vez sí, fue elegido el candidato apoyado por los musulmanes a través de la creación de la asociación Sabati 2012. Desde 2009 los actores islámicos sintieron el apoyo y la capacidad de ser escuchados por la población, y concretaron la creación de un movimiento político-religioso para influir en la política, desde el juego político institucionalizado.

\section{La creación del movimiento político-religioso Sabati 2012}

Como parte del fenómeno observado de la irrupción en política de los líderes y de las tendencias islámicas, surge el movimiento Sabati 2012. Esta joven asociación tiene un claro componente 
político-religioso y en ella participan mayoritariamente jóvenes musulmanes ${ }^{7}$ con una dilatada trayectoria en el activismo islámico, que han materializado lo que muchos líderes islámicos expresaban en público: que ante el derrumbe contemplado de la política, puesto en evidencia por la crisis institucional que arrastraba el Gobierno de Turé y que acabó en el golpe de Estado de marzo de 2012, los musulmanes debían movilizarse para "salvar al país". La opción escogida por los jóvenes musulmanes fue la participación en los procesos electorales que iban a tener lugar.

Sabati 2012 (prosperar - reafirmar su fe, en lengua bambara) se define como un movimiento para la promoción de los valores religiosos y sociales. Su fecha de creación, el año 2012, guarda relación con la celebración de las elecciones presidenciales que iban a tener lugar en abril de ese año (estatutos y reglamento interior de Sabati, 2014). Estas elecciones cerraban un ciclo político en la medida en que, finalizados los dos mandatos que el presidente Amadú Tumani Turé (2002-2012) había disfrutado, por ley no podía volver a presentarse a la elección. El presidente Turé, que no formaba parte de ningún partido, había gobernado con el apoyo de una coalición de partidos que apoyaron su candidatura independiente en el Parlamento y en las anteriores elecciones presidenciales, pero el balance de su gestión del país al cabo de ocho años era bastante criticado. Durante sus mandatos la población no había observado ninguna mejora en la obtención de servicios básicos y había percibido un encarecimiento de la vida, mientras que los casos de corrupción en el Gobierno y la administración eran constantes. Asimismo, la tensión política en el norte del país había empeorado y el estallido de una nueva rebelión armada era inminente.

A las deficiencias en el gobierno y a la dinámica política de gobierno en solitario en la que la oposición no tenía ningún papel ${ }^{8}$, pues todas las decisiones se tomaban en torno al "consenso" político (Baudais y Chauzal, 2006), se añadía la pésima administración de las regiones del norte y la gestión del conflicto abierto con las comunidades tuareg y árabes que históricamente han reclamado la formación de un Estado en el norte, el llamado "Azawad" . La plataforma Sabati

\footnotetext{
${ }^{7}$ Por lo general, la aplicación término "joven" a personas es bastante amplia. Se suele atribuir en contraposición a los "ancianos" que serían aquellos que ya tienen una edad avanzada y una amplia experiencia vital y laboral. El resto del serían los jóvenes, por lo que encontramos dentro de esa definición a personas entre 16-18 años y 40-45 años.

${ }^{8}$ Después de dos décadas de democracia y multipartidismo el contexto político se ha caracterizado, en primer lugar, por la debilidad de la oposición al presidente y al ejecutivo. Los grupos sociales y políticos opuestos han tenido una escasa representación en la Asamblea Nacional a pesar de la diversidad de partidos políticos representados en dicha cámara. El presidente Turé obtuvo el apoyo de la gran mayoría de los grupos políticos para gobernar, participando algunos de ellos en los ejecutivos formados por Turé. Una pequeña minoría de diputados de signo cercano al marxismo se consideraron como opositores, no obstante, sin obtener dicho reconocimiento. El estatuto de la oposición y la conformación de un "Jefe de la oposición", que ofrece un reconocimiento en la Asamblea y la posibilidad de obtener financiación de los presupuestos generales, no se ha establecido hasta la legislatura de 20132018. En segundo lugar, huelga decir que, a pesar de la gran diversidad de partidos políticos registrados, cerca de 145, la gran mayoría de ellos no tiene base social de relevancia ni una ideología concreta que los distinga entre ellos, siendo el factor personalista el elemento más importante.

${ }^{9}$ El norte de Mali es escenario de un conflicto entre parte de la comunidad tuareg y árabe y el Estado maliense, así como entre las comunidades tuareg y el resto de comunidades norteñas. El conflicto ha conocido varios episodios de violencia desde la independencia del país (1962-1964, 1990-1996, 2006-2009), el último, la rebelión que estalló en enero de 2012 en la que los grupos tuareg lograron expulsar a la administración maliense de las tres regiones del norte (Kidal, Gao y Tombuctú). En los enfrentamientos con el Estado maliense participaron otros grupos armados autodenominados yihadistas presentes en la región, como Al Qaeda en el Magreb Islámico (AQMI), el Movimiento por la Unicidad y la Yihad en África del Oeste (MUYAO), y el grupo armado islamista, Ansar Din. En abril de 2012, los rebeldes tuareg declararon la independencia unilateral del "Azawad", pero fueron dispersados, al igual que los grupos yihadistas, por una operación militar internacional liderada por Francia en enero de 2013. La pésima administración de las regiones del norte por los Gobiernos de Turé y su incapacidad para contener el estallido de la rebelión en 2012 y las ofensivas armadas de los rebeldes y yihadistas hicieron caer en desgracia al presidente. Su deposición mediante 82
} 
2012, que ya se había puesto en pie para las elecciones de 2012, puso en marcha sus actividades para las elecciones presidenciales de 2013.

El movimiento Sabati 2012 se creó como una plataforma de jóvenes musulmanes comprometidos con el islam y la defensa de sus valores. Lejos de ser un movimiento únicamente integrado por eruditos o personalidades islámicas, entre los fundadores también figuraron jóvenes profesionales de la empresa y del activismo islámico, como Mussa Bubacar Bah y Fodié N’Diaye, que en nuestros respectivos encuentros nos relataron que para fundar el movimiento se unieron con otras personas de diferentes perfiles, como médicos e intelectuales, muchos de ellos francófonos y procedentes de la escuela pública ${ }^{10}$. Al contrario de lo que podría pensarse por tratarse de un movimiento de naturaleza política con referencias islámicas, los fundadores e integrantes no provienen exclusivamente de ámbitos religiosos como podría ser la medersa o vinculados exclusivamente a asociaciones religiosas. Es frecuente también encontrar la opinión de que la creación de la organización fue impulsada por el presidente del Alto Consejo Islámico de Mali (ACIM), el imam Mahmud Dicko, que es considerado como máxima autoridad y representante del islam en el país. Esta afirmación se basa, en parte, por la cercanía de algunos de los miembros fundadores de Sabati 2012 con el presidente Dicko, como por ejemplo el presidente del movimiento y miembro del ACIM, Mussa Bubacar Bah.

El imam Dicko, sin embargo, ha negado cualquier protagonismo en la creación del movimiento y ha trasladado la idea de la fundación a una serie de jóvenes musulmanes a los que, como afirma, conoce y reconoce tener una relación cercana con alguno de ellos ${ }^{11}$. La acusación de que la organización fue fundada por Dicko y de que es un instrumento de su política debe ser enmarcada en la animosidad con que ciertos sectores islámicos y laicos de la sociedad maliense ven la popularidad y las acciones del presidente del ACIM, que se caracteriza por ejercer una gran influencia política y social en todo tipo de asuntos desde su designación en la presidencia del organismo musulmán en 2008. En el más extremo de los casos, para algunos críticos de la asociación, Sabati 2012 sería el brazo político y juvenil del presidente del ACIM. Además, esta organización es tildada de ser el brazo político del salafismo en Mali por la vinculación de algunos de sus impulsores y miembros con el reformismo salafí, tendencia con la que los detractores identifican también a Mahmud Dicko.

La fundación de Sabati 2012, según uno de sus fundadores, N’Dula Thiam, tiene que ver con el ámbito religioso en primer lugar. La reflexión que dio paso a su creación tuvo lugar en el seno de una asociación de estudiantes y antiguos estudiantes musulmanes, la LIEMA (Ligue islamique des étudiants du Mali) ${ }^{12}$, asociación islámica ampliamente implantada en los institutos de enseñanza secundaria y en las universidades de todo el país para la puesta en valor del islam en el espacio público educativo. En segundo lugar, la asociación se fundó para apoyar una alternativa que creyeron adecuada para el gobierno del país, máxime tras la crisis iniciada en 2012 luego del golpe

un golpe de Estado militar en marzo de 2012 no fue mal vista por la población, que no demostró demasiado entusiasmo en defender su mantenimiento a la cabeza del Estado.

${ }^{10}$ Entrevista con N. Thiam, miembro de Sabati 2012 y diputado del partido RPM, 9 de diciembre de 2014, Bamako; entrevista con F. N'Diaye, miembro de Sabati 2012, 28 de marzo de 2016, Bamako.

${ }^{11}$ Entrevista con M. Dicko, imam y presidente del ACIM, 5 de noviembre de 2014, Bamako.

${ }^{12}$ Entrevista con N. Thiam, diciembre de 2014, Bamako. 
de Estado que dejó sin un rumbo claro a la política maliense ${ }^{13}$. Para Thiam la organización nació como consecuencia directa de la caída en desgracia de las políticas del expresidente Amadú Tumani Turé y la confusión en torno a la alternativa de Gobierno para las elecciones de 2012.

En Sabati 2012 la referencia al islam está presente como elemento muy importante también en el proceso de toma de decisiones. El islam vincula a los miembros de la organización entre sí - la mayoría de los miembros, según afirman, son militantes de organizaciones islámicas, aunque con una trayectoria educativa y profesional diversa- pero, además, los guía para tomar las buenas decisiones basadas en el islam, en lo que consideran que debe de ser un musulmán, que representa los valores necesarios actualmente para ser no solo una buena persona, sino también un buen político. Asimismo, el objetivo de la fundación del movimiento era la demanda organizada de ciertas reivindicaciones para la mejora de ciertos aspectos del país, entre ellos algunos relacionados con el islam, como analizaremos más adelante.

La situación había acumulado anteriormente agravios para los musulmanes como el episodio de la aprobación del Código de familia y de las personas en 2009, que fue interpretado por los líderes musulmanes, aunque no exclusivamente, en clave de traición suprema hacia los valores religiosos e identitarios de la sociedad maliense ${ }^{14}$. Identificadas las elites gobernantes como responsables de la aprobación en la Asamblea Nacional de un Código profundamente contrario a los valores tradicionales y religiosos de la sociedad maliense, los jóvenes musulmanes creyeron que era posible un cambio de gobernantes a través de la sensibilización de sus correligionarios musulmanes. La gran exaltación popular y el encuadramiento de las masas en las calles de Bamako en los años 2009 y 2010 por los líderes islámicos en oposición a la aprobación del Código de la familia y de las personas reforzó la idea de que la movilización popular a partir de los líderes religiosos y por motivaciones referentes a la identidad religiosa y los valores de la sociedad, podía tener consecuencias de tipo político.

En efecto, las grandes movilizaciones populares obligaron al presidente Tumani Turé a ordenar una revisión del Código de familia y de las personas y a conformar una nueva comisión de redacción en el que los líderes islámicos tuvieron un peso determinante, en detrimento de los sectores de la sociedad civil laicos (Koné, 2015). Así, como algunos entrevistados del movimiento Sabati 2012 y otros líderes islámicos nos trasladaron, los acontecimientos en torno al episodio del Código de familia y de las personas hicieron tomar conciencia a los sectores islámicos sobre su gran capacidad de movilización y su influencia sobre las decisiones políticas, así como sobre la necesidad de sustituir a la clase política gobernante por otra más proclive a los valores religiosos de la sociedad maliense. Por tanto, tomaba forma la idea de que en los procesos electorales siguientes había que impedir que algunas de las personalidades al frente del país volviesen a controlar los resortes del Estado, personalidades a las que consideraban proclives a la introducción

\footnotetext{
${ }^{13}$ Entrevista con N. Thiam, diciembre de 2014, Bamako.

${ }^{14}$ Este episodio es uno de las piedras angulares del desplazamiento del interés de los líderes islámicos y de las personas comprometidas con el islam hacia la política. Es uno de los episodios más recurrentes a la hora de justificar la interferencia del islam en la política maliense. El nuevo Código, impulsado por el gobierno del expresidente Tumani Turé y aprobado por la Asamblea Nacional con cláusulas progresistas en el ámbito de la mujer, fue juzgado como inadecuado y no en consonancia con la sociedad por amplios sectores de la sociedad maliense. El descontento popular fue capitalizado por los líderes islámicos que se pusieron a la cabeza del malestar y demostraron una gran capacidad de movilizar a la población. Un análisis más detallado de esta cuestión puede encontrarse en Soares, 2009, Thiriot, 2010 y Koné, 2015.
} 
por ley de modificaciones "ajenas al país" similares al proyecto del Código de familia y como responsables de la desgracia del norte y el desgobierno del país ${ }^{15}$.

Un miembro de Sabati 2012, M. W., imam originario de Segú (centro sur del país), apuntó también a la influencia en la creación del movimiento que tuvieron las revueltas en los países del norte de África, conocidas como "Primavera árabe", y, sobre todo, los buenos resultados electorales de las agrupaciones islamistas y la integración de éstos en las estructuras de poder en países como Túnez o Egipto (Planet y Hernando de Larramendi, 2013) ${ }^{16}$. Si el modelo de estas agrupaciones islamistas o partidos no es atractivo para ellos según una opinión generalizada, sí veían en estos partidos el modelo en cuanto a la posibilidad de movilización de los musulmanes en un entorno libre y democrático, como el que, como algunos apuntan, Mali disfruta desde hace dos décadas ${ }^{17}$. Bubacar Bah aseguró que la posición de muchos jóvenes musulmanes, educados y formados en la "escuela occidental" les permitía tener una buena preparación y la capacidad de mediar y entender las aspiraciones de la población, una población abandonada por sus dirigentes desde hace varios años. Por tanto, los jóvenes musulmanes en el origen de Sabati 2012 se veían obligados a participar en política o a inmiscuirse en ella para ejercer de mediadores entre la población y la política y así evitar un desbordamiento hacia los extremos, que, analizado en clave religiosa, serían un extremo laico y un extremo religioso, cualquiera de los dos igual de peligroso para las aspiraciones de los malienses ${ }^{18}$.

\section{La participación del movimiento en las elecciones presidenciales del año 2013}

Sabati 2012 nació con tres ejes sobre los que trabajar y que, según su presidente, guiaron las decisiones del movimiento: el respeto a los valores religiosos, el respeto a los valores identitarios de la nación maliense y el respeto al entorno social ${ }^{19}$. Algunos de los miembros de Sabati 2012 son imames, y la justificación para dar el paso a la política, ya sea de la tendencia islámica que sea, es que el islam y la nación siempre han estado ligados, y por tanto, el islam no se puede

\footnotetext{
${ }^{15}$ Los datos de las escasas encuestas realizadas en Bamako (FES, 2012) y en resto del país (Coulibaly, 2014) arrojan datos que indican que los encuestados responsabilizaron en los primeros lugares a la clase política. La "falta de patriotismo de los líderes", la "debilidad del Estado" o “incompetencia de la clase política” aparecieron en los 4 primeros puestos en 2012 (Coulibaly, 2014), mientras que la "forma de gestionar las rebeliones del norte" y la "debilidad de la clase política" aparece en la segunda encuesta (FES, 2012). Por tanto, los síntomas de la desilusión en la política percibidos por el activismo islámico parecían coincidir con los síntomas generales de la población.

${ }^{16}$ La mezcla de la religión y la política en Mali no es algo que haga unanimidad entre la población. Y mucho menos el salto del islam a la política. Una gran proporción de la población lo ve con recelo. Ante la pregunta de si la religión debería de mezclarse con la política, una gran mayoría, entre el $70 \%$ y el $80 \%$ de los encuestados afirman que la religión debe de ser algo personal y no debería de mezclarse con la política (Hatløy y Coulibaly, 2015; Hatløy et al., 2015). Una forma directa de participar en política por los líderes musulmanes parece que no es apreciada como lo es en otros países donde existen partidos islamistas, y son modelos rechazados por la población encuestada y por las opiniones recogidas ante líderes religiosos. Otras formas de participar menos directas parecen que sí son apreciadas.

${ }^{17}$ Entrevista con M. W., marzo de 2016, Bamako.

${ }^{18}$ Entrevista con M. B. Bah, presidente de Sabati 2012, 10 de diciembre de 2014, Bamako.

${ }^{19}$ En realidad, la diferencia entre los tres principios no es muy clara, ya que vienen a ser más o menos lo mismo, haciendo hincapié en el respeto de los valores que ellos consideran como de la sociedad desde una óptica religiosa (entrevista con M. B. Bah, diciembre de 2014, Bamako).
} 
despreocupar de los asuntos de gestión del Estado ${ }^{20}$. Con estos principios la organización se creó, entre otros, con el objetivo de incidir en los siguientes procesos electorales, especialmente en el proceso que por el sistema semi-presidencial por el que se rige Mali tiene más importancia para el devenir del Estado, es decir, las elecciones presidenciales ${ }^{21}$. Como ya hemos dicho, las elecciones presidenciales se suspendieron tras el golpe de Estado del 22 de marzo 2012 aunque finalmente pudieron organizarse con la ayuda de la comunidad internacional, en julio y agosto de $2013^{22}$.

28 candidatos tuvieron la oportunidad de concurrir en la contienda electoral, la mayoría de ellos sin opciones claras de obtener un resultado aceptable y mucho menos de pasar a la segunda ronda según el sistema de elección presidencial maliense ${ }^{23}$. El movimiento Sabati 2012 deseaba incidir en la elección presidencial y para ello habían decidido apoyar a un candidato para que lograse la victoria. Innegablemente el mensaje de Sabati 2012 coincidía con el de ciertos líderes musulmanes que, no obstante, no se decantaron abiertamente por un candidato por sus responsabilidades en asociaciones, aunque sí veían con buenos ojos que otros hicieran pasar su mensaje ${ }^{24}$. Además de dicho apoyo, tenían la convicción de que desde el islam podrían ser escuchados por una audiencia más amplia que desde una tribuna política -por el evidente descrédito de los políticos en ese momento-. Los fundadores del movimiento consideraban que cualquier mensaje emitido por la clase dirigente y la clase política no iba a ser acogido por la población, crítica con las acciones, valores y principios de éstos. En cambio, los miembros de Sabati reconocían que el mensaje difundido por los religiosos sí sería al menos escuchado y, quizá, adoptado por la población maliense, ya sea religiosa o no, y, por lo tanto, aprovecharon esa coyuntura para incidir en las elecciones $\mathrm{y}$, como argumentaron, para indirectamente ejercer una mejora de la democracia.

En declaraciones en nuestra entrevista, el presidente de la asociación Bubacar Bah hizo hincapié en que ciertos valores y prescripciones islámicas tienen que ver con la mejora de la democracia y el respeto de los derechos humanos, y que, según él, el mensaje irradiado desde el exterior o desde la clase política no es aceptado tan fácilmente por la población como lo es desde las mezquitas, puesto que imames y líderes religiosos disfrutan de una confianza notable por parte de la población. Con la convicción de que lo islámico puede ser una referencia para una considerable mejora de los asuntos públicos, los miembros fundadores de Sabati decidieron emprender un proceso de selección entre los numerosos candidatos a la presidencia, para dilucidar quién de ellos merecía el apoyo de su organización, y, potencialmente, del de los musulmanes a los que Sabati aspiraba a movilizar.

Según lo manifestado por miembros del movimiento en las entrevistas realizadas, fueron siete los candidatos a la jefatura del Estado que fueron elegidos inicialmente en el movimiento para

\footnotetext{
${ }^{20}$ Una visión más que enfatiza que los religiosos deben de cumplir un papel para con el Estado, aunque sin tener un papel protagonista en primera línea como supondría la creación de un partido (entrevista con M.W., marzo de 2016, Bamako; entrevista con A. B., miembro de Sabati 2012, 24 de marzo de 2016, Bamako).

${ }^{21}$ Art. 9. 5. de los Estatutos del movimiento Sabati 2012.

${ }^{22}$ Durante este proceso electoral estuve acreditado como observador independiente, gracias al proyecto OPEMAM del Taller de Estudios Internacionales Mediterráneos (TEIM) de la Universidad Autónoma de Madrid.

${ }^{23}$ El sistema de elección presidencial maliense es un sistema de segunda ronda con circunscripción única. Si en una primera ronda ninguno de los candidatos ha obtenido un resultado mayor del $50 \%$ de los votos, los dos candidatos con mayor número de votos pasan a la segunda ronda.

${ }^{24}$ Es necesario puntualizar que el apoyo a Sabati 2012 no se ejerció desde todos los sectores islámicos. El movimiento no representa la voz y el respaldo de toda la comunidad de líderes islámicos hacia ciertos políticos. 
seleccionar entre ellos al candidato final ${ }^{25}$. La fórmula para poder discernir qué candidato merecía el apoyo de Sabati fue mediante entrevistas entre el candidato y miembros del movimiento ${ }^{26}$. Las entrevistas tuvieron lugar en la sede del ACIM, a falta de una sede del movimiento - lo que para algunos refuerza la conexión entre el presidente del ACIM y la plataforma-, y tuvieron el formato de un cuestionario sobre cuestiones políticas y sociales sobre el que se desarrollaron largas horas de entrevista, con el objetivo de dilucidar quién era merecedor del apoyo de los musulmanes para "sacar adelante este país" 27.

Las consultas dieron como resultado que el apoyo sería para el candidato Ibrahim Bubacar Keita, del partido Rassemblement Pour le Mali (RPM) ${ }^{28}$, antiguo primer ministro y expresidente de la Asamblea Nacional. Según los entrevistados, Keita era la persona que "encarnaba el cambio" que el país necesitaba después de la crisis política y securitaria en la que se había visto sumido. El candidato escogido era el "hombre fuerte" necesario para solventar los problemas y desafíos que el país debía afrontar ${ }^{29}$. En última instancia, también consideraron que Keita era la persona más adecuada para hacer efectiva sus demandas de promoción de los valores religiosos y morales de la sociedad (memorándum de Sabati, 2013), aunque no estaban de acuerdo con el conjunto de las tesis del partido al que pertenece Keita ${ }^{30}$. No era la primera vez que Keita era apoyado por los musulmanes en un proceso electoral. Durante las elecciones presidenciales de 2002, el candidato del RPM fue apoyado por una serie de líderes religiosos en torno al Collectif des Associations Musulmanes, no sin división entre ellos, dentro de la agrupación Espoir 2002 que encabezaba ${ }^{31}$. En dichos comicios, Keita alcanzó la tercera posición en la primera ronda y resultó eliminado. En la segunda ronda, la victoria fue para Amadú Tumani Turé.

\footnotetext{
${ }^{25}$ Los candidatos eran: Sumana Sacko, Muntaga Tall, Modibo Sidibé, Sheij Modibo Diarra, Ibrahim Bubacar Keita, Mussa Mara y Chogel Maiga. Según Bubacar Bah, Keita no estaba en la primera lista de seleccionados porque no había acudido a la reunión (entrevista con M. B. Bah, diciembre de 2014, Bamako).

${ }^{26}$ Entrevista con N. Thiam, diciembre de 2014, Bamako; entrevista con M. Kimbiri, periodista y miembro del ACIM, 4 de diciembre de 2014, Bamako.

${ }^{27}$ Que los candidatos a las elecciones presidenciales, incluido el propio Keita, acudieran a la reunión solicitada por la joven asociación Sabati 2012 demuestra el interés de los políticos en granjearse el apoyo de dicho movimiento, seguramente como un posible recurso adicional para tener mayores posibilidades de movilización del electorado, en este caso del electorado que se considera musulmán, y obtener un buen resultado en los comicios. En cambio, el candidato Cissé, que era uno de los candidatos potencialmente presidenciables y que no fue llamado por Sabati, denunció en la campaña electoral la intromisión de la religión en el juego electoral y el apoyo del movimiento a Keita (Correau, 2013). Las reacciones del electorado a la decisión de Sabati 2012 de apoyar un candidato observadas en Bamako durante la campaña electoral fueron heterogéneas, desde la celebración de dicho respaldo a la desconfianza por la intromisión percibida del islam en los comicios. Los sectores más laicistas y pro occidentales veían en la creación de Sabati 2012 y su participación en las elecciones una intromisión de la religión en política con el resultado de una posible mayor presencia de la religión en el Estado y un peligro para la laicidad. Por tanto, el elemento religioso se convirtió en un factor más en la contienda electoral de los candidatos y del debate político de la campaña electoral, generando reacciones heterogéneas en el electorado y los candidatos.

${ }^{28}$ El partido RPM es creado tras la escisión de Keita del partido ADEMA-PASJ en el año 2001. El partido forma parte de la Internacional Socialista desde el año 2003.

${ }^{29}$ Entrevista con M. Kimbiri, diciembre de 2014, Bamako.

${ }^{30}$ Entrevista con L. Djiguiné, miembro de Sabati 2012 y diputado en la Asamblea Nacional, 29 de marzo de 2016 , Bamako.

${ }^{31}$ Entrevista con M. Kimbiri, diciembre de 2014, Bamako.
} 
Una vez elegido el candidato, Sabati 2012 emprendió actividades en todo el país para hacer campaña electoral por el candidato Keita. Para ello algunos de sus miembros hicieron campaña en ciudades y pueblos, y, como algunos entrevistados explicaron, haciendo de la mezquita un foco de irradiación del mensaje cuando no también lugar de alojamiento de los miembros del grupo ${ }^{32}$. Durante su gira por el país, los miembros del movimiento recorrieron mezquitas y zauiyas, visitaron a sheijs e imames indistintamente de la tendencia islámica practicada con la voluntad de crear estructuras en las regiones y los cercles y que tanto ellos como sus fieles votasen al candidato Keita en las elecciones presidenciales. Según nos explicaron los responsables, durante la campaña electoral y las giras por las diferentes regiones pudieron comprender las preocupaciones de las poblaciones musulmanas $y$, tras haber estado en contacto con los líderes religiosos locales, llegaron a algunas conclusiones que luego se materializaron en parte de los principales puntos que argumentan para participar en las elecciones (memorándum de Sabati, 2013).

En cuanto a la desconfianza frente a la clase política, los miembros de Sabati la consideran el mayor problema de las casi dos décadas y media de democracia maliense. Las elites políticas habían basculado hacia posiciones poco claras, perdiendo la confianza de la población. Para remediar esta situación y "fortalecer la democracia”, el filtro que ellos aplicarían a los candidatos permitiría al elector tener una mejor referencia sobre a quién votar, $y$, por la confianza que irradian los religiosos, ese candidato tendría menos posibilidades de ser corrupto y trabajaría contra las injusticias.

Cabe hacer hincapié en que los líderes religiosos disfrutaban de una amplia confianza por parte de la población maliense. Tanto las observaciones y testimonios recogidos sobre el terreno, como las encuestas existentes, así lo demuestran con claridad. Este estamento destaca sobre otros en cuanto a la confianza que depositan los malienses ${ }^{33}$. Este prestigio permitiría salvar la desconfianza en los políticos, en un momento de crisis en el país en el que una amplia abstención electoral hubiera generado efectos catastróficos en el sistema y en la salida de la crisis.

El compromiso del movimiento Sabati 2012 con las reglas del juego democrático era pleno según manifestaban sus responsables. Para ellos, la democracia es una competición entre partidos y candidatos que hacen la política y que dirigen el país, y son necesarios para que el sistema democrático funcione. El aporte de la plataforma sería el fortalecimiento de los candidatos y, por tanto, de los partidos, para así mejorar la calidad democrática del país ${ }^{34}$. Sabati 2012 consideraba como otro factor que ha contribuido al desgobierno del país la injerencia de organismos o potencias extranjeras en la política y en la sociedad maliense. Por ello planteaban que un buen político es aquel que no deja que desde el exterior se influya en su actuación, -aunque Mali participe de ellos como miembro o sea firmante de convenios internacionales-, ya sea para desarrollar una legislación nueva o para tomar decisiones que no se consideren acordes con los valores de la sociedad maliense. En ese caso, el islam sería un seguro ante cierta posible deriva del Jefe del Estado.

\footnotetext{
${ }^{32}$ Entrevista con M. B. Bah, diciembre de 2014, Bamako.

${ }^{33}$ Las encuestas de Hatløy, Coulibaly y Liu (2015), Coulibaly y Diarra (2015) y Hatløy y Coulibaly (2015) arrojan una alta confianza de la población encuestada en los líderes religiosos, con solo una proporción cercana al 15\% que no confía en ellos y los ve como corruptos. Los estamentos que menos confianza generan eran los partidos políticos, la justicia y la policía.

${ }^{34}$ Entrevista con M. B. Bah, diciembre de 2014, Bamako. 88
} 
El islam era un elemento central en la definición del movimiento Sabati 2012, de sus actividades y de sus objetivos. Si la mayoría de sus integrantes activos y fundadores provienen del mundo asociativo religioso o se consideran a sí mismo religiosos, el programa que reivindicaban para el candidato al que apoyaban también tenía que ver con distintos ámbitos y aspectos de la religión en Mali y los debates sociales abiertos. Entre ellos se encontraban la preservación de la moralidad de la sociedad (adaptar el contenido audiovisual de la televisión a los "valores socioculturales del país", aplicar la legislación contra bares y lugares de ocio, prohibir la publicidad de tabaco y alcohol y "criminalizar" la homosexualidad) y la reforma de las relaciones entre el Estado y el islam (impulsar la formación de imames y predicadores, implicar a los religiosos en la organización del Hach, ampliar la financiación de estructuras religiosas por los partidos políticos, instaurar como festivo el día de la Achoura, el mantenimiento del Ministerio de Asuntos Religiosos y del Culto e integrar los valores socioculturales a la gobernanza) $)^{35}$.

Por tanto, en contra de las predicciones de muchos convencidos laicistas tanto dentro como fuera de Mali $^{36}$, el apoyo de Sabati 2012 y, por ende, el apoyo de una parte de los musulmanes a Keita, no era a cambio de la implantación de un programa islamista ni islámico, ni exigía la promoción de la práctica del islam o su proselitismo ${ }^{37}$ sino el compromiso de tomar decisiones que implicaran la mejora de las condiciones de los líderes islámicos y la regulación del islam, así como reconsiderar la laicidad en el Estado acorde con el aparente incremento de la presencia del islam en la esfera pública. Como algunos de los entrevistados apuntan, el político Keita se mostraba como un musulmán piadoso (como muchos otros personajes políticos en Mali) y expresaba ideas de respeto hacia la religión y hacia los valores de la sociedad. El candidato, por otro lado, es reconocido abiertamente como un laico ${ }^{38}$, aun así se muestra públicamente como un buen creyente. En cualquier caso, el candidato Keita era capaz de presentarse como un buen musulmán con el fin de movilizar al electorado religioso. En sus mítines y actos de campaña, durante la campaña electoral, Keita comenzaba sus intervenciones con versículos del Corán y trufaba sus discursos con un característico "inshallah", reconocido por todos. La utilización de cierto lenguaje religioso era apreciada como un guiño a los líderes islámicos y a los votantes musulmanes, y creó cierta inquietud entre algunos sectores laicistas que sospechaban de la posibilidad de una menor separación entre Estado y religión si Keita triunfaba en las elecciones. Como ya había sucedido en el año 2002, Keita parecía ser favorable a la consideración de tales demandas y a no ir en contra

\footnotetext{
${ }^{35}$ En lo reflejado en el memorándum presentado a los candidatos, Sabati 2012 ponía atención en la intención de crear y llevar a cabo una política musulmana por parte del Estado que tratase los temas islámicos, aunque no exclusivamente. La lista de demandas estaba trufada de varias propuestas en los ámbitos de la seguridad, el ámbito social, de la educación, de justicia, agrícola y de gobernanza. Algunos autores (Holder, 2013) sugieren que esas son las demandas que desde hace tiempo reclama en ACIM. La mayoría de las demandas no se han hecho efectivas o se han satisfecho a medias durante la primera mitad del mandato de Keita, mientras que algunas sí se han realizado (memorándum de Sabati, 2013).

${ }^{36}$ Después del apoyo abierto de una parte de los musulmanes, los sectores más laicistas y en defensa de los derechos de las mujeres, así como las cancillerías extranjeras, pusieron el foco en las posibles decisiones que el gobierno Keita pudiera ejercer en adelante hacia los religiosos a cambio de los apoyos ofrecidos a través de Sabati durante su elección en asuntos relacionados con el respeto de la laicidad del Estado y los derechos de la mujer (entrevista con M. D., mujer miembro de asociación civil en defensa de los derechos humanos y derechos de la mujer, 12 de diciembre de 2014, Bamako).

${ }^{37}$ Entrevista con F. N'Diaye, marzo de 2016, Bamako.

${ }^{38}$ Entrevista con Dicko, noviembre de 2014, Bamako.
}

REIM № 23 (Diciembre 2017) ISSN: $1887-4460$ 
de los colectivos religiosos. En palabras de uno de los fundadores de Sabati 2012, N'Diaye ${ }^{39}$, era preferible apoyar "alguien que nos dejara trabajar, que a alguien que no".

Para poder llevar a cabo sus actividades y la estructuración del movimiento, los miembros fundadores y cercanos a Sabati 2012 el movimiento se financió principalmente con fondos de los propios miembros del movimiento $\mathrm{y}$, especialmente, con recursos provenientes de un líder islámico. Los entrevistados afirman que la campaña para el candidato Keita se financió gracias a los fondos donados por el sheij tiyaní de Nioro du Sahel, el Sheij Hamallah. Tras la decisión de apoyar al candidato Keita, el líder islámico - así como personas de su entorno- anunció que destinaba la suma de 10 millones de CFAs (unos 15000 euros) para la campaña electoral del candidato del $\mathrm{RPM}^{40}$.

El sobrino de Hamallah también contribuyó con la cantidad de 3 millones de CFAs (alrededor de 4 500 euros) a la campaña de Sabati $2012^{41}$. Según su presidente, el movimiento completó sus ingresos con el apoyo de otros sectores, como comerciantes, funcionarios y abogados, así como miembros de la ejecutiva de Sabati 2012 que también contribuyeron con grandes sumas de dinero en el proyecto de apoyo a la elección del candidato Keita. Asimismo, otra forma de financiarse ha sido la cotización mensual de los miembros de la organización. La financiación exterior es uno de los puntos sobre los que los entrevistados hicieron hincapié, insistiendo en que no han recibido fondos del extranjero ${ }^{42}$.

Los dirigentes del movimiento calcularon que la capacidad de movilización en torno al candidato Keita era de un $10 \%$ o un $15 \%$ que mejoraría respecto a la movilización natural que el propio candidato podría obtener sin la ayuda de Sabati 2012. Sin explicar en qué basaban esos cálculos, afirmaron que ese fue el peso que tuvieron durante su elección, aportación sin la cual no hubiera podido obtener dicha contundente victoria electoral. Para ello promovieron que las mezquitas fuesen un lugar de difusión del mensaje y de su consigna de voto. La existencia de cientos de mezquitas en Bamako y en el resto del país suponía una gran potencialidad para hacer campaña entre la población ${ }^{43}$. Su uso con un objetivo político no fue del agrado de todos los imames, llegándose a dar incluso la negativa de los fieles para darle ese uso a la mezquita ${ }^{44}$. También este uso generó desconfianza entre los sectores de oposición al candidato Keita.

Ibrahim Bubacar Keita ganó en la segunda ronda con un apoyo del 77,62\% de los votos, con más de 2 millones de votos (Nievas, 2013). Una vez aupado a la Jefatura del Estado, al parecer la relación del movimiento con el presidente Keita ha sido ambigua. El propio diputado N'Dula

\footnotetext{
${ }^{39}$ Entrevista con N’Diaye, marzo de 2016, Bamako,

${ }^{40}$ El papel jugado por el sheij de Nioro du Sahel en la plataforma no ha podido ser analizado porque no hemos podido acceder a nadie de su entorno debido a nuestras limitaciones de movimiento, restringido al distrito de Bamako, ya que el líder y la mayoría de sus seguidores están en Nioro, en la región fronteriza con Mauritania. En cualquier caso, todas las decisiones parecen haber sido informadas al sheij con antelación, como la elección de Keita como el candidato a respaldar (entrevista con Kimbiri, diciembre de 2014, Bamako).

${ }^{41}$ Entrevista con M. B. Bah, diciembre de 2014, Bamako.

${ }^{42}$ Entrevista con N. Thiam, diciembre de 2014, Bamako.

${ }^{43}$ No existe en Mali un censo del número de mezquitas y oratorios en la ciudad de Bamako. Estete es uno de los proyectos que el gobierno de Keita pretendía impulsar como parte de su política musulmana. Hemos encontrado una referencia al número de mezquitas en la capital maliense a principios de 1980 en un estudio que cuantificó y cartografió las medersas y mezquitas, realizado por Fodé Doumbia y publicado en 1987. El estudio cuantificó más de 200 mezquitas (Doumbia, 1987, citado en Brenner, 2001: 197).

${ }^{44}$ Entrevista con M. B. Bah, diciembre de 2014, Bamako. 
Thiam, miembro de Sabati 2012 y elegido por el partido RPM en las elecciones legislativas de 2013, reconocía que el presidente no conocía quiénes eran un año después de su elección ${ }^{45}$, dando a entender que no estaban en la esfera de poder del presidente y que éste no tenía en cuenta a la asociación como un pilar más de la red de apoyo y gestión de su Gobierno ${ }^{46}$. En el entorno próximo al presidente Keita parecía que no había religiosos, ni miembros de Sabati 2012 ni del ACIM. La consulta del presidente con la organización, quizá contemplada por Sabati tras contribuir a su elección - y que desvelaría uno de sus objetivos implícitos-, no había tenido lugar con la frecuencia que al grupo le hubiera gustado.

Las declaraciones a la prensa del presidente del movimiento, Mussa Bubacar Bah, dejaban también entrever cierto descontento con el gobierno de Keita y el nivel de interlocución entre las partes (Diallo et al., 2013). El descontento no iba dirigido contra el presidente como un ejercicio de oposición -ya que afirmaron que el compromiso de apoyo era para la duración del mandato presidencial de 5 años $^{47}$-, sino que se interpreta más bien como una llamada de atención a cómo debería de hacer las cosas. A parte del mantenimiento del Ministerio de Asuntos del Culto como Ministerio delegado del Ministerio de la Administración Territorial y su promoción a principios de 2014 a Ministerio al mismo nivel que el resto de Ministerios, el resto de reivindicaciones plasmadas en el memorándum de Sabati no habían sido satisfechas. Como admitieron el presidente de Sabati 2012 en prensa (Keïta, 2014) y miembros destacados del movimiento en nuestras conversaciones: "que se les consulte y escuche". Los miembros de Sabati 2012 consideran que ellos podían hablar y opinar libremente al Jefe del Estado y que, al estar vinculados a sus bases, podrían identificar las preocupaciones de la población ${ }^{48}$. Con el paso del tiempo, y el aumento de las dificultades en la gestión del país, el presidente y su Gobierno se habían reunido aparentemente con mayor frecuencia con los religiosos, también con los miembros de Sabati 2012, estableciendo lo que podría calificarse como una suerte de alianza. Si en el primer año de su mandato, pareció que el presidente Keita gobernó manteniendo a distancia a los religiosos, a lo que algunos mostraron su descontento como hemos mencionado antes, a partir del segundo año de su mandato se observó un mayor acercamiento e interlocución entre las partes (Segbedji, 2015).

\footnotetext{
${ }^{45}$ Entrevista con N. Thiam, diciembre de 2014, Bamako.

${ }^{46}$ Según recalca Bubacar Bah (entrevista con M. B. Bah, diciembre de 2014, Bamako), no se permite que ningún miembro de Sabati tengo un cargo de responsabilidad en un partido político. En el caso de los diputados apoyados por Sabati, algunos de ellos miembros activos del movimiento, y su integración en partidos políticos, se explica que éstos no ejercen ningún cargo orgánico dentro de ellos y que simplemente se adscriben a ellos con el fin de reforzar el partido.

${ }^{47}$ Entrevista con L. Djiguiné, marzo de 2016, Bamako.

${ }^{48}$ Entrevista con N. Thiam, diciembre de 2014, Bamako.
} 


\section{Conclusiones}

Aunque la formación de iniciativas como la de Sabati 2012 parece que tiene un interés, por decirlo de un modo sencillo, de islamización de la política o de islamización de la sociedad, al ser preguntados sobre este aspecto hay una opinión unánime en los impulsores de la iniciativa al señalar que la situación de Mali no era la de aquellos países donde ha emergido el islamismo, como Túnez, Argelia o Egipto. Todos ellos expresan un rechazo a los movimientos políticos islamistas que buscan conquistar el poder. En general la opinión de los responsables religiosos de este movimiento político-religioso sobre los grupos islamistas es negativa, poniendo de relieve que no han sido la solución para sus países sino que generaron más inestabilidad (en referencia a la anulación de la victoria del Frente Islámico de Salvación en Argelia en 1991 y la posterior inestabilidad en el país, y la irrupción en el poder de los Hermanos Musulmanes en Egipto en 2012) y que una alternativa así no es lo que necesita actualmente Mali. La impresión generalizada de estos actores islámicos interesados en la política es que la sociedad maliense no está preparada para ello y además, el concepto de laicidad (una laicidad ${ }^{49}$ que acepte el pluralismo religioso en el espacio público creando una esfera islámica) es defendido por casi todos los principales líderes políticos y no está en cuestión. Asimismo, la creación de un partido político de corte islámico (si es que se lograsen sortear las trabas legales que significa la formulación de la laicidad en la Constitución y en la ley de partidos políticos) o la candidatura política a la Jefatura del Estado de un personaje religioso, vendría a certificar una relación directa de la religión con la política que plantea dudas sobre la acogida que tendría entre la población.

Hemos podido observar que el paso a la política a partir del campo islámico por el momento no es mayoritario, sino que se trata de algo minoritario pero organizado, y que las razones esgrimidas por los que ahora están desarrollando actividad política en ese sentido no se encuentran en la voluntad de querer obtener el poder a partir de un proyecto islámico (lo que sería un proyecto islamista). Aunque a lo largo de la historia democrática de Mali se han dado casos en los que un marabú sufí ha pasado a la política formal como alcalde o se señalaba que algún parlamentario provenía de los círculos religiosos, no era algo tan frecuente ni eran casos que contaban con una organización detrás (Leininger, 2010) ${ }^{50}$. Sin embargo, lo encontrado en la actualidad es una tendencia a esa participación más consolidada. Se puede afirmar que la participación en política de individuos relevantes y movimientos con referencias islámicas, así como líderes islámicos, ya no es solo una posibilidad entre otras sino un recorrido que ha comenzado a ser transitado en el Mali de la última década como demuestra la trayectoria de Sabati 2012 en las elecciones presidenciales de 2013.

La fórmula adoptada es el acompañamiento de los políticos en las elecciones a través del apoyo en la campaña electoral y la movilización del electorado por medio de los mensajes a su favor desde las mezquitas, es decir, tratar de capitalizar su ascendencia entre la sociedad con la intención de influir posteriormente en decisiones políticas o ser incorporados en las consultas políticas en temas de su interés. Así, la participación política del islam, encuadrada en un marco laico y una fuerte presencia e intervención internacional en el país liderada por Francia, no está motivada por

\footnotetext{
${ }^{49}$ Mali heredó el concepto de laicidad de la antigua potencia colonial, Francia. De ella se inspiró para establecer en sus textos fundacionales la laicidad del país en referencia a que el Estado no se ocuparía de los aspectos religiosos de su población ni se pronunciaría sobre la religión, tratándola desde un punto de vista neutral.

${ }^{50}$ Como por ejemplo la elección en 2009 de H. Sankaré en la alcaldía de Uankoro, cercle (que se podría traducir como provincia) de Bankass (Mopti, centro del país) o la llegada a la Asamblea Nacional de figuras religiosas como Y. Haidara, muqaddam sufí.
}

92 
la toma del poder y la imposición de un modelo distinto sino que es impulsada principalmente por la reivindicación de algunos sectores islámicos - tanto sufíes como reformistas salafíes- de que el Estado procure mayor atención a los asuntos religiosos del país y la regulación del campo islámico, así como por la voluntad de una mayor extensión - o al menos de que el ejecutivo no vaya en contra- de la moralidad y del respeto a los valores religiosos en la sociedad y entre la clase política como elemento necesario, según estos sectores religiosos, para cumplir con el contrato social y mejorar la gobernanza. La exploración por algunos sectores islámicos de la tendencia a interesarse en política y participar en ella tendría que ver, en primer lugar, con la expansión de la esfera islámica durante las más de dos décadas y media de democracia en Mali, que ha situado a algunos líderes islámicos en una situación de gran visibilidad y de respeto dentro de la sociedad maliense, y en segundo lugar, estaría relacionada con las consecuencias de la incapacidad de las élites políticas de la última década en afrontar los desafíos del país y la evidente desconexión existente entre estas élites y la población, ilustrada con el episodio del Código de la familia y de las personas.

En el origen de la nueva presencia en política estaría el debate para la aprobación del Código de familia y de las personas en 2009, que generó una amplia reacción de los sectores populares liderados por los personajes religiosos que los movilizaron, y la iniciativa promovida por ciertos líderes de participación en los procesos electorales. Las movilizaciones populares pueden ser consideradas una demostración de fuerza del estamento religioso en una suerte de pulso político con el Estado en el proceso de contrarreforma del Código de la familia y de las personas. El apoyo que recibió de la sociedad maliense hizo que saliera claramente fortalecido en detrimento de otros sectores de la sociedad civil laica (Villalón, 2010; Koné, 2015) y contribuyó al descrédito de la clase gobernante a ojos de la población. La idea del salto a la política formal no tuvo su génesis exclusivamente en esa época, pues ya había sido una posibilidad explorada por parte de los religiosos a principios de los años 2000, pero la gran exaltación popular y el encuadramiento de las masas por los líderes islámicos en oposición al Código de la familia y de las personas reforzó dicha idea, que finalmente se materializó en las elecciones presidenciales del año 2013 a través del apoyo al candidato Keita. Así, los actores islámicos impulsaron el movimiento Sabati para hacer realidad la participación política y materializar su peso en la sociedad, aunque tal acontecimiento no fue del agrado unánime de la población ${ }^{51}$ ni de los actores laicos y pro occidentales ${ }^{52}$, lo que ha generado, por el momento, cierta polémica y debate en el país.

\footnotetext{
${ }^{51}$ De los resultados de unas encuestas realizadas antes y después de las elecciones presidenciales de 2013 por la delegación en Bamako de la fundación Friedrich Ebert Stiftung, se desprende que la población de Bamako no era muy favorable a ese activismo de los religiosos en las elecciones ( $60 \%$ consideraba negativo o muy negativo el papel de los religiosos en las elecciones en mayo de 2013 y un porcentaje similar en septiembre de 2013) (FES, 2012; 2013).

${ }^{52}$ Entrevista con A. D., responsable de asociación civil, 20 de noviembre de 2014, Bamako.
} 


\section{Bibliografía}

BAUDAIS, Virginie y CHAUZAL, Grégory (2006): “Les partis politiques et l'«indépendance partisane» d'Amadou Toumani Touré", Politique africaine, n104, pp. 61-80, https://doi.org/10.3917/polaf.104.0061

BRENNER, Louis (2001): Controlling Knowledge. Religion, Power, and Schooling in a West African Muslim Society, Bloomington, Ind., Indiana University Press.

CORREAU, Laurent (2013): Soumaïla Cissé: "Je pense être beaucoup plus moderne que mon adversaire", Radio France International, disponible en http://www.rfi.fr/afrique/20130808-malisoumaila-cisse-presidentielle-ibk [consultado el 2 de diciembre de 2017].

CORMACK, David. (2005): "An African Vortex: Islamism in Sub-Saharan Africa", Occasional Paper Series, 4, Center for Security Policy.

COULIBALY, Massa (2014): "Popular Perceptions of the Causes and Consequences of the Conflict in Mali", Afrobarometer Policy Paper, 10, disponible en http://afrobarometer.org/publications/pp10popular-perceptions-causes-and-consequences-conflict-mali-0 [Consultado el 14 de diciembre de 2016].

COULIBALY, Massa y DIARRA, Djénéba (2015): "Religion et politique au Mali", Afrobaromètre. Informe original de Afrobaromètre, publicado bajo la organización GREAT, disponible en: http://www.greatmali.net/data/Article 55 3.pdf [Consultado el 28 de febrero de 2017].

DIALLO, Moussa, SAMBA, Sogodogo y DRAMÉ, Ousmane (2013): "Le nouveau gouvernement vu par Moussa Boubacar Ba, président de Sabati 2012: «Ces gens ne m'inspirent pas le changement "", Le Républicain, disponible en: http://news.abamako.com/h/25493.html [Consultado el 23 de agosto de 2016].

DICKSON, David (2005): Political Islam in Sub-Saharan Africa. The Need for a New Researchand Diplomatic Agenda, United States Institute of Peace, Special Report, no140.

DOUMBIA, Fodé (1987): “Les mosquées à Bamako", Jamana: Revue culturelle malienne, no13, pp. 31-35.

FRIEDRICH EBERT STIFTUNG (2012): Enquête d'opinion. Que pensent les Maliens?., disponible en: http://www.fes-mali.org/index.php/mali-metre/25-malimetre/88-malimetre-1 [Consultado el 15 de febrero de 2017].

FRIEDRICH EBERT STIFTUNG (2013): Mali-mètre 2. Enquête d'opinion. Que pensent les Maliens?, disponible en: http://www.fes-mali.org/index.php/mali-metre/25-malimetre/91-malimetre-2 [Consultado el 15 de febrero de 2017].

HATL $\varnothing$ Y, Anne y COULIBALY, Mamadou (2015): "Les questions ethniques et religieuses", Les GREAT cahiers, n51, Fafo, disponible en: http://www.greatmali.net/data/Article\%20 51.pdf [Consultado el 22 de febrero de 2017].

HATL $\varnothing$ Y, Anne, COULIBALY, Mamadou y LIU, Jing (2015): "Réorientation religieuse au sud du Mali - Rapport de statistiques", Rapport Fafo 2015, n14, disponible en web: http://www.fafo.no/index.php/nb/zoo-publikasjoner/fafo-rapporter/item/reorientationreligieuse-au-sud-du-mali-rapport-de-statistiques [Consultado el 23 de febrero de 2017]. HOLDER, Gilles (2009): L'islam, nouvel espace public en Afrique, París, Karthala.

HOLDER. Gilles (2013): "Un pays musulman en quête d'Etat-nation”, en GONIN, Patrick, KOTLOK, Nathalie y PÉROUSE DE MONTCLOS, Marc Antoine (eds.): La tragédie malienne, Paris, Vendémiaire, pp. 135-160.

KEÏTA, Youssouf (2014): “Moussa Boubacar Bah, Président de Sabati 2012 : « Le Président IBK ne consulte pas et n'écoute pas... tout le monde a été mis en quarantaine»", Le Républicain, disponible en: http://www.maliweb.net/politique/moussa-boubacar-bah-president-sabati-2012- 
president-ibk-consulte-pas-necoute-pas-monde-ete-mis-en-quarantaine-428562.html [Consultado el 28 de agosto de 2016].

KONÉ, Ousmane (2015): Le controverse autour du code des personnes et de la famille au mali: enjeux et strategies des actuer, (Tesis doctoral), Université de Montréal, Montreal. Disponible en: https://papyrus.bib.umontreal.ca/xmlui/bitstream/handle/1866/13576/Koné Ousmane 2015 th ese.pdf?sequence=2\&isAllowed=y\%3E [Consultado el 28 de septiembre de 2016].

KÜNKLER, Mirjam y LEININGER, Julia (2009): "The Multi-faceted Role of Religious Actors in Democratization Processes: Empirical Evidence from Five Young Democracies", Democratization, no16, pp. 1058-1092, https://doi.org/10.1080/13510340903271746.

LAREMONT, Ricardo y GREGORIAN, Hrach (2006): "Political Islam in West Africa and the Sahel”, Military Review, n으, 1, ProQuest Research Library, pp. 27-36.

LEININGER, Julia (2010): "The Diverse Role of Muslim Actors in Mali's Democratic Consolidation: Fostering Plurality to Inhibiting Strong State Institutions", paper prepared for the Workshop on Religious Actors in Democratization Processes: Evidence from the five Muslim Democracies to be held in Princeton University February 4-5, 2010, pp. 1-30.

LEININGER, Julia (2016): "It's Institutions, Not Theology! Muslim Actors' Influence on Democratization in Mali", Politics and Religion, no9, pp. 815-842, https://doi.org/10.1017/S1755048316000584

MILES, William F. S. (2007): Political Islam in West Africa: State-Society Relations Transformed. Boulder y Londres, Lynner Rienner.

MOUVEMENT SABATI 2012 (2014): Statuts et réglement intérieur.

MOUVEMENT SABATI 2012 (2013): Mémorandum du Mouvement Sabati 2012 aux candidats, disponible en: http://www.maliweb.net/societe/memorandum-du-mouvement-sabati-2012-auxcandidats-158754.html [Consultado el 12 de septiembre 2015]

NIEVAS BULLEJOS, David (2013): “Ficha electoral: Elecciones presidenciales Mali 2013", OPEMAM. OTAYEK, Réné y SOARES, Benjamin Frankiln (2007): "Islam and Muslim Politics in Africa", en . SOARES, Benjamin F. y OTAYEK, Réné (eds.): Islam and Muslim Politics in Africa, Nueva York, Palgrave Macmillan US, pp. 1-24.

PEW RESEARCH CENTER (2011): Muslim-Majority Countries - The Future of the Global Muslim Population, disponible en: http://www.pewforum.org/interactives/muslim-population-graphic/ [Consultado el 10 de enero de 2017].

PLANET, Ana Isabel y HERNANDO DE LARRAMENDI, Miguel (2013): "Spain and Islamist Movements: from the Victory of the FIS to the Arab Spring", en VIDINO, L. (ed.): The West And The Muslim Brotherhood After The Arab Spring. Filadelfia; Dubadi: Al Mesbar Studies \& Research Centre; The Foreign Policy Research Institute, pp. 114-130.

SEGBEDJI, Bruno D. (2015): "IBK reçoit l'imam Mahmoud Dicko et le président de " Sabati 2012 " pour réchauffer leurs rapports", Le Républicain, disponible en: http://www.maliweb.net/politique/ibk-recoit-limam-mahmoud-dicko-et-le-president-de-sabati2012-pour-rechauffer-leurs-rapports-808382.html [Consultado el 8 de noviembre de 2016].

SCHULZ, Dorothea (2003): “'Charisma and brotherhood' revisited: Mass-mediated forms of spirituality in urban Mali", Journal of Religion in Africa, no33, 2, pp. 146-171, https://doi.org/10.1163/15700660360703123

SOARES, Benjamin F. (2009) "L'islam à l'ère néolibérale", en OTAYEK, Réné y SOARES, Benjamin F. (eds.). Islam, état et societé en Afrique, París, Khartala, pp. 411- 433. 
THIRIOT, Celine (2010): "Islam et espace public au Mali. Une société civile très engagée" en DARBON, Dominique, OTAYEK, Réne y SARDAN, Pierre O. (eds.). Alterité, et identité, itineraires croisés. Mélanges oferts à Christian Coulon, Bruselas, Bruylant, pp. 213-243.

VILLALÓN, Leonardo (2010): "From Argument to Negotiation: Constructing Democracy in African Muslim Contexts", Comparative Politics, n42, 4, pp. 375-393, https://doi.org/10.5129/001041510X12911363509954

WING, Susana (2008): Constructing Democracy in Transitioning Societies of Africa Constitutionalism and Deliberation in Mali. Nueva York: Palgrave Macmillan US. 